\title{
VOCES MIGRANTES FRENTE A LA VIOLENCIA DE GÉNERO': UNA INVESTIGACIÓN PARA LA ACCIÓN EN SAN FRANCISCO (EEUU) ${ }^{2}$
}

\author{
Migrant Voices Confronting Gender-based Violence: A Research for Action \\ in San Francisco (USA)
}

MARÍA PILAR TUdela-VÁZQUEZ ${ }^{3}$

\begin{abstract}
Resumen
Introducción: La intersección entre la violencia de género y el hecho migratorio, produce situaciones de vulnerabilidad concretas que dificultan el acceso de muchas mujeres a los recursos disponibles. Objetivos:1) Partir de las redes asociativas para atender a los procesos de formulación de necesidades y recomendaciones comunitarias. 2) Proponer la Investigación Acción Participativa (IAP), donde la trabajadora social desarrolle un papel de dinamización, como herramienta metodológica de enriquecimiento de la intervención social. Material, Métodos y Resultados: IAP que la autora desarrolló en la organización Mujeres Unidas y Activas, California (EEUU). Participaron 148 mujeres que incorporaron sus experiencias, necesidades y recomendaciones para la mejora de la calidad de los servicios sociales disponibles. La investigación se estructuró en 3 partes. A) Fase preinvestigación, B) Fase de Diagnostico Comunitario, y C) Fase de Herramientas para la Acción. Resultados: Esta investigación permitió visibilizar el potencial de los espacios comunitarios como ámbitos de intervención en donde las mujeres sobrevivientes se insertan como agentes de cambio. Discusión: La IAP nos permite, como profesionales de la intervención social, implicarnos en espacios de convergencia que permitan generar nuevas respuestas y/o mejorar las existentes.
\end{abstract}

Palabras Clave: Violencia de Género, Migraciones, Intervención Social Comunitaria, Investigación Acción Participativa.

\begin{abstract}
Introduction: The intersection between genderbased violence and migration produces specific situations of vulnerability that hinder the access of women to available social services. Objectives: 1) To take associative networks as the starting point to address the processes of needs assessment and community recommendations regarding violence against women. 2) To propose Participatory Action Research (PAR), where the social worker develops a facilitator role, as a methodological tool to enrich social intervention. Materials, Methods and Results: PAR developed by the author at the organization of Mujeres Unidas y Activas, California (USA). 148 women participated by incorporating their personal experiences, needs and recommendations to improve the quality of the social services available for assistance in cases of intimate partner violence against women. The research was structured in three phases. Phase A: Pre- research, Phase B: Community Diagnosis and Phase C: Tools for Action. Results: This research highlighted the potential of community spaces as areas of intervention where women survivors are inserted as agents of change. Discussion: PAR allows us, as social intervention professionals, to get involved in areas of convergence which are critical to generate new answers and / or improve existing ones.
\end{abstract}

Keywords: Gender-based violence, Migrations, Community Social Intervention, Participatory Action Research.

1. Como veremos más adelante, la organización define el problema de la violencia de género o machista, como violencia doméstica. Por coherencia con el proceso metodológico que aquí se presenta, la denominación de tan amplia problemática como «violencia doméstica» se mantendrá a través del texto, una vez presentada la definición que se desarrolló.

2. Los datos que aquí se presentan corresponden al trabajo de campo desarrollado en San Francisco y Oakland, entre 2006 y 2010 para la tesis doctoral «La Organización de Nosotras: Procesos de ciudadanía a partir de experiencias de 'ilegalidad' en Estados Unidos. Aprendizajes con Mujeres Unidas y Activas» dirigida por Prof. Carmen Gregorio Gil. Dpto. Antropología Social. Universidad de Granada.

3. Universidad de Granada. Dpto. Antropología Social. Facultad de Filosofía y Letras. Campus Universitario de Cartuja. 18071, Granada. E-mail: mtudela@ugr.es 


\section{Introducción}

La presente investigación se inserta en el marco de los trabajos que cuestionan la premisa de la violencia de género como un problema expresado de manera uniforme en la vida de las mujeres independientemente de la sociedad en la que vivan, orientación sexual, cultura, clase social o nivel educativo. Partimos de que las formas que adopta esta violencia y sus significados dependen de las relaciones sociales y contextos culturales en donde toma forma (Merry, 2009; Yoshihama, 1999), y por lo tanto, del papel que determinados ejes estructurales desarrollan a la hora de situar a las mujeres en espacios de vulnerabilidad localizados (Menjívar y Salcido, 2002).

Estas aportaciones abogan por incorporar en el estudio para la intervención y prevención de la violencia de género una perspectiva estructural que atienda a la diversidad de experiencias de las mujeres, partiendo de un análisis interseccional del papel que juegan la clase social, la nacionalidad, el género y la raza (Sokoloff y Dupont, 2005; Rivera, 1994; Crenshaw, 1991). Desde una perspectiva estructural, el problema de la violencia de género expresada en la vida de las mujeres migrantes no puede ser analizada como una cuestión únicamente relacionada con la distribución diferenciada y desigual de poder entre hombres y mujeres (Bhuyan y Senturia, 2005). Debemos complejizar los niveles de desigualdad y adoptar una visión integrada del papel que desempeñan otros ejes, como el racismo, el etnocentrismo, los privilegios de clase o el heterosexismo en su intersección con el género (Villalon, 2010). Esta forma de análisis nos permite explicar las razones de muchos de los factores identificados como relevantes en la vida de mujeres migrantes en relaciones violentas. Así, el estatus migratorio, la falta de redes sociales de apoyo, la precariedad laboral, y/o la falta de medios económicos (Alencar-Rodrigues, Cantera, y Strey, 2013; Fundación Cepaim, 2013; Montañez y Moyano, 2006), dejan de ser realidades estáticas e inamovibles, y se presentan como posibles ejes de intervención social en donde el Trabajo Social desarrolla un papel clave en el impulso de procesos organizativos comunitarios.

La prevalencia de la intervención individualizada en el problema de la violencia contra las mujeres, en detrimento de actuaciones a nivel grupal y comunitario, ha sido identificada como uno de los obstáculos para poder apoyar a las mujeres que buscan transformar su situación de subordinación (AlcazarCampos, 2013). La atención individualizada como única respuesta, contradice aquellas propuestas que desde el Trabajo Social, presentan la construcción de relaciones de apoyo mutuo y el trabajo grupal, como elementos clave para la adquisición de herramientas vitales de empoderamiento, como son la auto percepción y definición positiva y el desarrollo de habilidades y nuevos 
conocimientos (Wood y Middleman, 1992). Otro de los obstáculos señalados corresponde a la excesiva confianza depositada en los sistemas de intervención legal, como la principal puerta de entrada para adquirir asistencia. Importantes trabajos desarrollados en relación a mujeres pobres que pertenecen a comunidades definidas por nociones de exclusión y criminalidad, basadas en estereotipos de raza, etnicidad y/ o experiencias de ilegalidad ${ }^{4}$, muestran la ambivalencia de estas mujeres a la hora de recurrir a la policía en un caso de violencia de género (Bhuyan y Senturia, 2005; Menjívar y Salcido, 2002; Rivera, 1994). La posibilidad de que las intervenciones estatales reproduzcan las vivencias de maltrato y desempoderamiento que están viviendo en sus relaciones de pareja, previene a muchas mujeres de acudir a estos servicios en primer lugar (Coker, 2006; Strang y Braithwaite, 2002).

Los espacios comunitarios han sido identificados como lugares clave desde los que poder llegar a mujeres reticentes a utilizar aquellos servicios sociales que implican a la policía y priorizan la separación familiar (Sokoloff y Dupont, 2005). La construcción y promoción de respuestas comunitarias orientadas al fortalecimiento del tejido social supone una importante contribución para el trabajo preventivo y de apoyo en casos de violencia contra las mujeres (Lee, 2013). Concretamente, Wood y Middleman (1992) han definido dos formas de intervención claves que en Trabajo Social podemos desarrollar en este contexto. Partiendo del trabajo en grupo, ambas autoras resaltan la promoción y desarrollo de oportunidades de aprendizaje que permitan a las participantes conectar sus experiencias individuales con condiciones estructurales. Este tipo de procesos permite construir sentimientos de colectividad, a la vez que potencia formulas positivas de redefinición subjetivas. En segundo lugar consideran como intervención clave, facilitar oportunidades para que las mujeres tomen la iniciativa en el uso de recursos propios y comunitarios para promover cambios vitales, "participando y construyendo redes comunitarias, enfrentando obstáculos organizativos, y comprometiéndose en acciones políticas» (Pinderhuges en Wood y Middleman, 1992, p. 88).

Un objetivo de este artículo es abogar por respuestas de prevención y atención en materia de violencia contra la mujer en la pareja que partan de intervenciones comunitarias promovidas por organizaciones de base (Minkler, 2005), en donde la trabajadora social desarrolle un papel de facilitación. Como profesionales de la intervención social, esta investigación supone un ejemplo del potencial dinamizador que tiene el situarnos en aquellos procesos

4. La ilegalidad se define como el espacio de reproducción social producido a partir de la desconexión entre la presencia física en un espacio dado y el reconocimiento del derecho a permanecer en dicho espacio (De Genova, 2005) 
en donde la participación viene promovida por propuestas organizativas como las que destacan Wood y Middleman.

Un segundo objetivo es incidir, a través de una experiencia práctica, en la aplicación de la Investigación Acción Participativa (IAP) como herramienta metodológica necesaria para el enriquecimiento de la intervención social y en general, para la construcción de conocimiento y práctica científica en Ciencias Sociales (Humphries, 2008; Greenwood y Levin, 2006; Ander-Egg, 2003).

En el contexto de intervención en salud pública y comunitaria, la IAP viene siendo reconocida como herramienta para la promoción de cambios a nivel comunitario y estructural (Minkler y Wallerstein, 2011; Minkler, 2005; Wallerstein, 2000). En el área de la violencia de género, y concretamente la violencia en la relación de pareja, existen algunos trabajos publicados que muestran el potencial de la IAP a la hora de acercarnos, desde diferentes puntos de vista, a las realidades de las mujeres migrantes (Sullivan, Bhuyan, Senturia, Shiu-Thornton, y Ciske, 2005). Así encontramos investigaciones que recogen: 1) percepciones de la violencia en la pareja de mujeres refugiadas procedentes de países en guerra (Bhuyan, Mell, Senturia, Sullivan, y ShiuThornton, 2005; Shiu-Thornton, Senturia, y Sullivan, 2005); 2) experiencias de mujeres migrantes indocumentadas a la hora de solicitar recursos legales para casos específicos de violencia en la pareja (Ingram et al., 2010); 3) evaluación y recomendaciones en relación a los programas y servicios sociales disponibles (Bhuyan y Senturia, 2005). La mayor parte de estos trabajos inciden en la IAP en tanto que herramienta de acercamiento y problematización de perspectivas unidimensionales a la hora de atender las experiencias concretas de mujeres migrantes. Al mismo tiempo, es necesario continuar profundizando en el objetivo de la acción misma de la investigación. Es decir, plantear desde dónde parte la necesidad de investigar, y qué consecuencias tiene el proceso de la IAP para las comunidades que se involucran en dicho proceso.

En la investigación aquí presentada, se entiende la participación como un proceso guiado de reflexión colectiva y de búsqueda conjunta de propuestas para el cambio, por parte de los grupos afectados por la problemática social en la que se quiere incidir, atendiendo al grado de implicación de las partes interesadas (Villasante y Montañés Serrano, 2000; Greenwood, Whyte, y Harkavy, 1993). Por lo tanto, involucra a las personas afectadas, entendidas como agentes de cambio, en todas las fases de la investigación, incluyendo el diseño, la implementación, análisis y divulgación de los resultados. La propuesta consiste en que el papel de los trabajadores sociales sea proveer de 
las herramientas metodológicas necesarias para abrir procesos colectivos de reflexión y búsqueda de propuestas para el cambio (Ioé, 1993).

El presente artículo dedica una parte importante al proceso de la investigación con el objetivo de reflejar la relevancia de las metodologías utilizadas. Estas permiten asegurar un alto nivel de implicación a lo largo de su implementación y se encuentran articuladas desde el compromiso de situar en el centro del problema de la violencia de género vivida por mujeres migrantes, las necesidades y propuestas elaboradas por las propias protagonistas. Así mismo, a través de la propuesta metodológica presentada se incide en la necesidad de que el motor del proceso esté conformado por una visión y definición de cambio compartida, que trascienda las necesidades individuales y promuevan una mayor capacidad para la prevención y respuesta al problema de la violencia contra la mujeres en el ámbito de las relaciones de pareja.

\section{Métodos y Materiales}

Este estudio se desarrolla en Estados Unidos, concretamente en las ciudades de San Francisco y Oakland, California, en donde trabajé durante seis años como trabajadora social y educadora en el Dpto.de Educación para la Salud Comunitaria (Casa CHE) de La Clínica de la Raza. Este trabajo me permitió conocer de primera mano tanto las limitaciones como las potencialidades de un sistema de servicios sociales diverso y descentralizado. Este contexto dista mucho del español en cuanto al papel social del Estado y desarrollo de provisiones públicas de protección social. Caracterizado por una larga tradición de provisión de servicios sociales y aplicación de programas de intervención social por parte de un complejo y diverso entramado de agentes privados con y sin ánimo de lucro. Estos entran en contacto con las instituciones gubernamentales a través de financiación de programas de ayuda específicos y/o de convocatorias para proyectos de intervención social.

Siendo el fortalecimiento del tejido asociativo uno de los objetivos de la promoción de la salud comunitaria, entré en contacto con numerosas organizaciones trabajando en el área. Una de estas organizaciones fue Mujeres Unidas y Activas (MUA), con más de veinte años de historia y que cuenta con la participación de más de 200 mujeres en Oakland y San Francisco.

El proceso de la Investigación Acción Participativa se desarrolló como se muestra en la tabla presentada a continuación: 
Tabla 1: Fases de la Investigación-Acción

\begin{tabular}{|l|}
\hline A) Fase Pre-investigación/Acción \\
\hline - Demanda que guía la intervención. \\
- Definición de objetivos generales \\
- Constitución de Grupo motor. \\
- Diseño de la Investigación y Proceso \\
\hline B) Fase del Diagnostico Comunitario y Resultados \\
\hline 1) Definición del Problema: ¿qué es la violencia doméstica? \\
2) Encuesta. \\
3) Análisis grupal de los resultados de la encuesta. \\
4) Compilación de primeros resultados. \\
5) Grupos de enfoque. \\
\hline C) Fase de Herramientas para la Acción \\
\hline 1) Elaboración de Recomendaciones \\
2) Plan de Acción: Definición y puesta en marcha. \\
- Identificación de agentes políticos y sociales (Directorio). \\
- Herramienta de Difusión. Informe \\
- Capacitación para presentaciones en público \\
- Equipos para dar presentaciones \\
- Priorización de interlocutores (listado) \\
3) Implementación- Evaluación-Devolución. \\
- Presentaciones y reuniones \\
- Recoger devolución \\
- Valorar su incorporación \\
- Definir próximos pasos \\
\hline
\end{tabular}

Fuente: Elaboración propia en base a IAP. San Francisco, California 2009

\section{A) Fase Pre-investigación/Acción. Identificación de Objetivos}

En el año de 2008, el Departamento del Estatus de la Mujer de San Francisco $^{5}$ financió programas locales en materia de violencia de género, para ser implementados a través de organizaciones sin ánimo de lucro, que proveyeran servicios en las siguientes áreas: teléfonos de asistencia, atención y

5. En 1998, San Francisco fue la primera ciudad de EEUU que adoptó los principios recogidos en UN Convention on the Elimination of All forms of Discrimination Against Women (CEDAW), a través del Dpto. del Estatus de la Mujer y, la ya existente, Comisión del Estatus de la Mujer. Ambos organismos, Comisión y Departamento, tomaron como referencia este marco legal transnacional de Derechos Humanos de las Mujeres, para el desarrollo de políticas y programas dirigidos a la mejora de la calidad de vida de mujeres y niñas residentes en la ciudad y Condado de San Francisco. En mi artículo «Levantando Nuestra Voz»: Traducciones Locales de los Derechos Humanos de las Mujeres» se describe el contexto político en el que se inscribe esta investigación (Tudela-Vázquez, 2013). 
acompañamiento, servicios legales, prevención y educación, casas de acogida y pisos tutelados. Una de las organizaciones beneficiarias fue MUA, la cual inició una campaña comunitaria cuyo objetivo era visibilizar los obstáculos a los que muchas de las mujeres de la organización se enfrentaban cuando buscaban ayuda para salir de una relación violenta. Siendo este el objetivo general, me invitaron a colaborar en el proceso de la campaña, aportando apoyo técnico en el desarrollo de una investigación participativa, cuya finalidad era conocer y recopilar las experiencias de Mujeres Latinas Inmigrantes ${ }^{6}$ en relación a la violencia de género, así como evaluar con ellas los dispositivos y servicios de atención disponibles. Una vez finalizada esta etapa elaboraríamos un informe con recomendaciones que sirviera de herramienta para entablar diálogo, tanto con organismos institucionales como con organizaciones sociales. Dadas las características de la intervención en la que este estudio se insertaba, esta campaña se llamó «Campaña de Derechos en Violencia Doméstica».

Además de los objetivos generales presentados, el grupo motor, formado por dos educadoras, dos coordinadoras de la organización, y yo como facilitadora técnica, identificamos tres objetivos adicionales: 1) Incorporar la evaluación de los propios programas de la organización durante el proceso de investigación; 2) Capacitar a las participantes en temáticas relacionadas con la investigación y 3) Fortalecer las relaciones de la organización con otras organizaciones. Así mismo, diseñamos las fases de la investigación, las técnicas a utilizar y un cronograma.

Las técnicas utilizadas fueron una combinación de tipo cuantitativo, fundamentalmente la encuesta, y de tipo cualitativo, como grupos de discusión y de enfoque. También llevamos a cabo la aplicación de la observación participante, y técnicas participativas orientadas a la promoción de la reflexión y desarrollo de propuestas colectivas, aportando al estudio una perspectiva dialéctica, la cual incorpora una noción de sujeto participante como productor de conocimiento colectivo e implica presentar claves dinamizadoras que provoquen la reflexión (Alberich Nistal, 2008; Villasante \& Montañés Serrano, 2000).

El principal criterio de selección de las participantes fue su pertenencia a algunos de los grupos de trabajo de la organización, como los grupos abiertos de reunión semanal y los comités de trabajo dedicados al área de intervención en violencia de género y al desarrollo de liderazgo comunitario. A partir de

6. Con estas tres palabras se autodefine la organización, de manera que situar «in» delante de migración es parte de la autodenominación de la asociación y de las mujeres que la conforman. Así será presentado cuando sea el caso, de lo contrario mi opción preferente es el término «migrante» 
este criterio, el número de participantes en cada una de las actividades que comprenden esta investigación, se determinó en base a la disponibilidad y voluntad de las propias mujeres.

En total participaron 148 mujeres, con edades comprendidas entre los 18 y los 55 años, procedentes en su mayoría de México, y en menor medida de El Salvador, Nicaragua, Guatemala, Perú y Uruguay. Residentes en el Área de la Bahía de San Francisco, concretamente en las ciudades de San Francisco, Oakland, Richmond, San Leandro y Hayward.

\section{B) Fase del Diagnóstico Comunitario}

El proceso de Diagnóstico Comunitario se construye sobre la base dialéctica de la propia Investigación-Acción. Cada una de las fases de la investigación se encuentra delimitada por los resultados que se hayan obtenido en la fase anterior, por las reflexiones colectivas derivadas del análisis de estos resultados, así como por las preguntas que hayan surgido y que definen la metodología a seguir en la siguiente fase.

1) Definición del problema: ¿qué es la violencia doméstica? A través de dos grupos de discusión, elaboramos la definición de «violencia doméstica» de

Tabla 2. Definición de Violencia doméstica. Mujeres Unidas y Activas, 2008

Mujeres Unidas y Activas define la Violencia Doméstica como una forma de conducta ejercida por una persona para mantener el poder y el control sobre otra persona. Incluye las siguientes características:

- La violencia doméstica ocurre dentro de la familia, es silenciosa, está escondida, y es mantenida por normas sociales y culturales, tales como el machismo.

- La violencia doméstica no es la única forma de abuso. Existe también el abuso emocional, sexual y financiero, incluyendo insultos, intimidación, amenazas y violación, los cuales forman parte de una relación abusiva.

- La violencia doméstica impacta y es impactada por otras formas de violencia, tales como la violencia política (p.e, el encarcelamiento de inmigrantes indocumentados); violencia comunitaria, (p.e. vecindarios con altos índices de criminalidad y escasos recursos), violencia económica (p.e, explotación de los trabajadores inmigrantes)

- Para las mujeres inmigrantes, las amenazas acerca del estatus migratorio son a menudo usadas por sus compañeros como una forma de ejercer poder y control.

- La violencia doméstica ocurre entre esposos, novios, parejas LGBTQ, adultos mayores y sus cuidadores, padres e hijos o familiares, prostitutas y sus clientes o tratantes, así como víctimas de persecución y tráfico humano.

- Aunque la violencia doméstica puede impactar a cualquiera, la mayoría de las víctimas son mujeres y niños.

Fuente: Elaboración propia en base a IAP. San Francisco (California), 2009

Alternativas. Cuadernos de Trabajo Social, 22, 2015, pp. 185-206 - ISSN 1133-0473 
Mujeres Unidas y Activas. Se desarrollaron dos sesiones, con un total de 16 participantes; cada sesión fue facilitada por dos personas del grupo motor y se desarrollaron de igual manera. Las preguntas guía para la facilitación fueron: ¿Que tipos o formas de violencia se dan en la violencia doméstica? ¿Cuáles son las diferencias entre la violencia doméstica y otras formas de violencia? ¿A quién/quienes afecta la violencia doméstica? ¿Cuáles son las raíces de la violencia doméstica? La Tabla 2 muestra la definición resultante de esta dinámica.

2) Encuesta: Se implementó una encuesta de 22 preguntas cerradas de respuesta múltiple, diseñada por el grupo motor, que se aplicó a las 148 mujeres participantes. Esta herramienta permitió adquirir información acerca de la problemática identificada, pero sobre todo fue un ejercicio exploratorio para el diseño de las técnicas cualitativas que se aplicarían posteriormente. Los objetivos de la encuesta fueron, entre otros, saber cuántas de las participantes habían vivido experiencias de violencia doméstica y de qué tipo, de acuerdo a la definición elaborada, a qué servicios habían acudido, si habían obtenido servicios en español y cómo valoraban su experiencia en la búsqueda de ayuda. Sistematizamos y agrupamos los datos obtenidos en base a las siguientes categorías: 1) Incidencia de violencia en la pareja, 2) Obstáculos que impiden buscar ayuda, 3) Acceso y Experiencias en relación a los Servicios Disponibles 4) Factores que promueven buscar ayuda.

3) Análisis grupal de los resultados de la encuesta. El objetivo fue analizar a través de grupos de discusión los datos de la encuesta. La sesión se desarrolló de manera paralela en ambas sedes de la organización. En Oakland participaron un total de 27 mujeres y en San Francisco, 37. Cada sesión duró dos horas y media aproximadamente.

4) Compilación de primeros resultados del Diagnóstico Comunitario. A continuación se presentan los datos cuantitativos obtenidos en combinación con algunas de las aportaciones que surgieron durante las sesiones de análisis grupal.

- Incidencia de violencia en la pareja. Los resultados mostraron que 8 de cada 10 mujeres (81\%) habían experimentado alguna forma de violencia. De aquellas mujeres que habían experimentado alguna forma de violencia, la violencia física y verbal fueron las más frecuentes. Ochenta y cinco por ciento (85\%) indicaron haber experimentado violencia verbal y casi las tres cuartas partes $(73 \%)$ experimentaron violencia física. Una de cada cuatro (26\%) experimentó violencia sexual. Una participante describió cómo la violencia se expresa de 
Figura 1. Porcentaje de mujeres que encontraron cada uno de los obstáculos para salir de la relación de violencia

\section{Obstáculos Identificados}

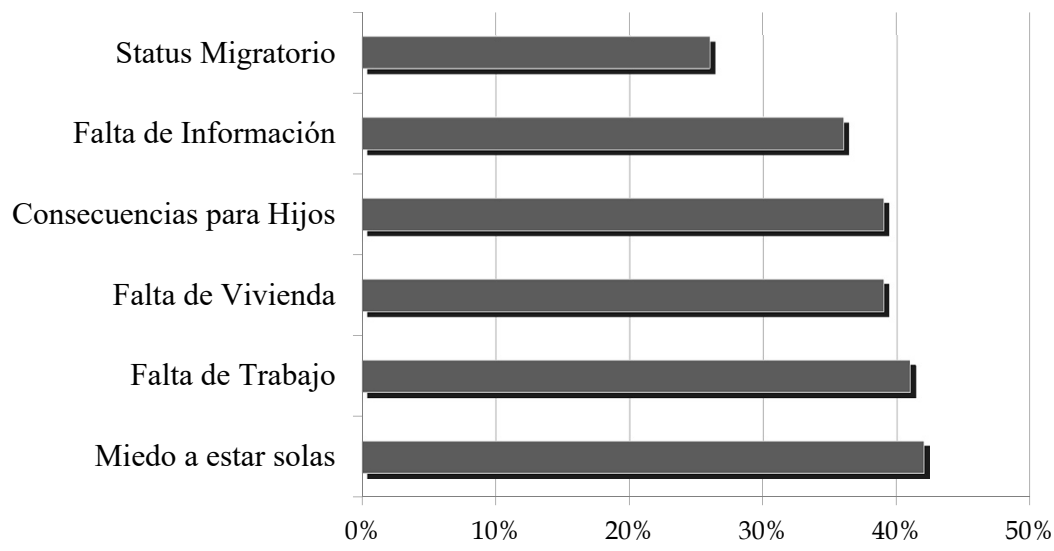

Fuente: MUA Encuesta. San Fancisco, 2009.

diferentes formas: «la violencia sexual es tanto el ser forzada a hacer el acto sexual como la humillación de tener que escuchar que una huele a esto o al otro».

- Obstáculos que impiden buscar ayuda. La Figura 1 presenta un resumen de los obstáculos identificados.

Como puede verse, los efectos negativos en los hijos fue una preocupación manifestada por muchas (39\%), y de manera particular en el caso de las casas de acogida. Una de las participantes (P1), en los grupos de análisis de la encuesta compartía su propia experiencia:

Tengo un niño de 17 años y una niña de 14, y por las edades que tienen te frenan porque no te los puedes llevar contigo. Entonces los exponen a otro trauma más grande porque estamos saliendo de un problema y pasar otro problema de que tienes que separarte de ellos. Muchas veces no sales de la situación por esto, o te vas a buscar ayuda en otro lado.

Como compartió otra participante (P2) otro obstáculo identificado está relacionado con el status migratorio: «muchas veces, una mujer es amenazada por su propio marido a ser denunciada a Inmigración, o a perder sus hijos si ella habla y él hace que la deporten». Una de cada 
Tabla 3: Experiencias de Servicios Contra la Violencia Doméstica

\begin{tabular}{|l|c|}
\hline \multicolumn{2}{|c|}{$\begin{array}{c}\text { Servicios de Violencia Doméstica } \\
\text { No Accedidos }\end{array}$} \\
\hline No acudieron a ningún servicio & $18 \%$ \\
\hline No fueron a una casa de acogida & $69 \%$ \\
\hline \multicolumn{2}{|c|}{ Experiencia en Servicios } \\
\hline La experiencia fue buena & $79 \%$ \\
\hline No fueron bien atendidas & $21 \%$ \\
\hline \multicolumn{2}{|c|}{ Acceso a traducción al español } \\
\hline Recibió traducción siempre & $54 \%$ \\
\hline $\begin{array}{l}\text { Recibió traducción usualmente o } \\
\text { Varias veces }\end{array}$ & $44 \%$ \\
\hline Nunca recibió el servicio & $2 \%$ \\
\hline \multicolumn{2}{|c|}{ Sentimiento de discriminación } \\
\hline No percibió discriminación & $67 \%$ \\
\hline Percibió discriminación & $33 \%$ \\
\hline \multicolumn{2}{|c|}{ Si experimento discriminación, que tipo } \\
\hline Por no hablar Ingles & $64 \%$ \\
\hline Por ser Latina & $48 \%$ \\
\hline
\end{tabular}

Fuente: MUA Encuesta. San Fancisco,2009.

4 mujeres (26\%) expresó que su status migratorio es un obstáculo para escapar de la violencia.

- Acceso y Experiencias en relación a los Servicios Disponibles. En la Tabla 3 pueden verse los principales resultados.

Casi la mitad de las participantes (44\%) recibieron servicios de traducción algunas veces o casi siempre. La otra mitad (54\%) dijeron haber recibido servicios de traducción en todo momento. Sin embargo, una participante (P3) comenta: «Hay lugares donde te ayudan por teléfono, donde sí hay traductor pero a veces cambian lo que tú estás diciendo. Lo que tú estás diciendo no es exactamente lo que ellos están traduciendo»

La atención psicológica (terapia), Grupos de Apoyo y Teléfonos de Asistencia fueron los servicios más utilizados. Más de la mitad (51\%) de las mujeres encuestadas eligió la terapia como recurso, y 
cuarenta y ocho por ciento (48\%) escogió grupos de apoyo. Una de cada cuatro (25\%) manifestó haber usado los teléfonos de asistencia. Una educadora (E1) explicaba: «reciben con más frecuencia servicios en español cuando acuden a servicios de terapia o grupos de apoyo. Además, estos servicios no incluyen separación familiar o llamar a la policía»

5) Grupos de Enfoque: Un resultado de la encuesta fue la infrautilización de los servicios legales e institucionales disponibles, y en especial las Casas de Acogida. Al mismo tiempo, la falta de vivienda fue identificada como uno de los obstáculos principales para salir de la violencia. Siete de cada 10 mujeres encuestadas no optaron por ir a una casa de acogida. De las mujeres que sí lo hicieron, cuarenta por ciento (41\%) definió la experiencia como «normal», quince por ciento (15\%) identificó la experiencia como «mala». Casi un cuarenta por ciento (39\%) abandonaron el programa manifestando que si estuvieran en la misma situación, no volverían a la casa de acogida.

Dinámica Grupo de Enfoque 1: En este grupo se exploraron, a partir de un guión de preguntas abiertas, las experiencias de mujeres que habían residido en casas de acogida. Participaron 10 mujeres, la mayoría procedentes de México, todas con un número de hijos entre 3 y 7 . Residieron en casas de acogida entre el 2003 y el 2007, y todas menos una completaron el programa de la casa de acogida.

Una de las preguntas fue en relación a los obstáculos que pueden intervenir a la hora de decidir ir a una Casa de Acogida. Las participantes identificaron las siguientes razones por las que consideraban que muchas mujeres no acuden a una Casa de Acogida.

- Preocupación por las consecuencias que tiene en los hijos e hijas salir de su casa y tener que ir a una casa de acogida.

- El aislamiento en el que la mujer se encuentra, sobre todo cuando acaba de llegar a Estados Unidos.

- La falta de información sobre los recursos disponibles y sobre sus derechos.

- Las malas experiencias de mujeres en Casas de Acogida, previene a otras de acudir a este recurso.

Además se compartieron las siguientes características que una casa de acogida debería de tener: 
- Personal que Hable Español en todo momento. Todas las participantes compartieron la barrera del idioma como un gran obstáculo.

- Atención para jóvenes, niñas y niños, en las casas de acogidas. Las participantes expresaron la necesidad de servicios psicológicos, programas educativos y espacios y normas que les permitan jugar.

- Apoyo para prevenir el maltrato entre las residentes y del personal a las residentes. Varias participantes reconocían que hay personal atento y dedicado a su labor. Al mismo tiempo compartían que no hay mecanismos para denunciar al personal que impone las reglas de forma desigual, y emplean el favoritismo.

- Apoyo para transitar los sistemas legales y de servicios sociales. Varias participantes expresaron no haber contado con toda la información que necesitaban para poder tener un plan de salida de la Casa de Acogida adecuado.

Casi todas las participantes recomendarían a una amiga y/o familiar ir a una casa de acogida en caso de violencia doméstica. Además de proveer un alojamiento, comida y seguridad, varias compartieron haber tenido el apoyo y la solidaridad que necesitaban, especialmente cuando las trabajadoras sociales las habían derivado a Mujeres Unidas y Activas.

Todas las participantes reconocían que ir a una casa de acogida puede ayudar a mejorar la vida, ofreciendo la oportunidad de buscar trabajo y ahorrar. Insistieron en la necesidad de que las Casas de Acogida estén en contacto con asociaciones comunitarias para asegurar que las mujeres se sientan seguras, apoyadas y con la información necesaria para poder tomar decisiones que las ayuden a salir adelante.

Dinámica Grupo de Enfoque 2: El objetivo fue explorar las causas por las que un número importante de encuestadas no acudieron a una casa de acogida. Participaron un total de 18 mujeres, la mayoría procedentes de México con un rango de edad entre los 20 y los 50 años, que no acudieron a una casa de acogida y eligieron buscar apoyo en sus redes cercanas o grupos de apoyo comunitarios. Casi el cuarenta por ciento (39\%) indicó que todavía vivían violencia con sus parejas cuando el grupo de enfoque se estaba desarrollando.

Al preguntar al grupo por qué no acudirían a una casa de acogida, las participantes identificaron los siguientes obstáculos:

- Las largas listas de espera para poder entrar a una casa de acogida.

- No tener suficiente apoyo en su idioma en el momento en que deciden dar el paso. 
- Tener hijos en edad adolescente que no pueden entrar en la casa de acogida

- No tener hijos, y por tanto menos posibilidades de entrar en una casa de acogida.

- Presentar casos de maltrato psicológico, los cuáles son considerados de menor riesgo que la violencia física y/o sexual.

Muchas mujeres identificaron la falta de información y de apoyo para utilizar los servicios de atención disponibles, el aislamiento, y las malas experiencias de otras mujeres en casas de acogida, como las razones principales por las que no eligieron este servicio. Este grupo también habló acerca de las ventajas y desventajas de buscar apoyo en redes cercanas y/o intentar salir sola de la relación violenta. Señalaron al grupo de apoyo de Mujeres Unidas y Activas como el lugar en donde estaban aprendiendo información útil para poder identificar «lo que no es normal» en una relación. Además en este grupo se compartió lo siguiente:

- Acudir a una casa de acogida y/o a la policía ayuda a que el caso legal de una mujer tenga más fuerza. El grupo discutió acerca de las ventajas que tiene hacer denuncias a la policía como tramite para fortalecer sus casos legales y acceder a otros recursos. También expresaron las dificultades por no hablar inglés, no tener papeles, el miedo a que te deporten y separen de los hijos e hijas, o no tener la información y estar aislada.

- Tener que irse de casa y cambiar el ritmo de vida de los hijos e hijas es una gran desventaja. Varias participantes reconocieron que, independientemente de a donde vayas, casa de acogida o a casa de familiares/amigos, tener que salir de tu casa junto con los hijos y ser la persona que tiene que cambiar de vida, es en sí un gran obstáculo. No tener trabajo y las consecuencias en la vida de los hijos e hijas, son los factores que más influyen a la hora de tomar esta decisión.

- Las redes familiares y/ amigos ayudan mucho cuando las relaciones familiares son saludables, pero también pueden ser un gran obstáculo. Las participantes reconocieron que si encuentran apoyo en las redes más cercanas, prefieren acudir a estas en primer lugar. La gran mayoría había llegado al grupo de apoyo de MUA de la mano de alguien que conocían. Al mismo tiempo, se compartieron experiencias de aislamiento y violencia fomentadas por el miedo y el chantaje en el ámbito de las redes familiares. 


\section{C) Fase de Herramientas para la Acción}

1. Elaboración de Recomendaciones. Concluido el diagnóstico comunitario, en el grupo motor preparamos la metodología con la que desarrollar las recomendaciones de la organización. Estas se desarrollaron en dos fases, protagonizadas por un total de 30 mujeres.

Definición de Recomendaciones: Recopilamos la información recogida y la repartimos entre las participantes para agrupar las recomendaciones que habían ido surgiendo en el Grupo de Enfoque 1. Se identificaron las siguientes categorías: 1) Idioma/Acceso a recursos, 2) Jóvenes y niños, 3) Empleados: Formación y Atención al usuario, 4) Calidad general de servicio, 5) Apoyo de la organización. A partir del análisis de los resultados del Grupo 2, se añadieron las siguientes categorías 6) Fortalecer los espacios comunitarios 7) Respetar los diferentes procesos de sanación. Criterios utilizados en la definición de recomendaciones: que fueran «posibles», es decir, que fueran alcanzables, potencialmente sostenibles, y que respondieran a cambios que beneficiaran a «grupos diferentes».

En total se desarrollaron 6 recomendaciones principales, que se presentan tal y como vienen recogidas en el informe:

- Fortalecer Redes de Apoyo Comunitarias. Las mujeres Latinas inmigrantes que están en una relación de violencia y maltrato necesitan encontrar información de servicios de apoyo en los lugares en donde ellas desarrollan su día a día y en su idioma.

- Incorporar a Mujeres acompañantes en los Servicios de apoyo y asistencia en Violencia Doméstica. MUA cuenta con sesenta mujeres que participan activamente en la organización y que han recibido el curso de capacitación para detección y asistencia de casos de violencia doméstica aprobado por el Estado de California (EC 1037.1). Los servicios sociales específicos para esta problemática enriquecerían y aumentarían su competencia cultural si incluyeran en sus programas a mujeres hispanohablantes capacitadas para dar servicios y responder a las necesidades de las mujeres inmigrantes.

- Construir y Ampliar Colaboraciones. MUA considera prioritario que se formalicen colaboraciones entre servicios comunitarios formados y dirigidos desde la comunidad inmigrante, con otros servicios como casas de acogida, servicios legales y jurídicos.

- Mejorar la Atención Profesional. Es necesario que los profesionales que trabajan en esta problemática estén capacitados, a nivel humano 
y profesional. Es importante que acompañen a las mujeres a lo largo de todo el proceso, ayudando a transitar complejos sistemas legales y de ayudas públicas, presentando en todo momento a la mujer cuáles son sus opciones y posibilidades.

- Respetar y Apoyar Diferentes Procesos de Sanación. Mujeres Unidas y Activas promueve a través de su modelo de trabajo respuestas colectivas para romper el aislamiento que la persona en violencia doméstica está viviendo, respetando en todo momento su proceso personal. Apoyar a mujeres que están pasando por experiencias por las que otras mujeres de la organización también han vivido, promueve la sanación de todas. Es en las respuestas colectivas y de solidaridad donde está la fuerza de sanación individual y comunitaria.

- Priorizar Fondos Económicos para Servicios y Organizaciones Comunitarias. En el momento de crisis económica actual, las familias con pocos recursos económicos están viviendo grandes dificultades que ponen en riesgo su calidad de vida. Ahora más que nunca es necesario fortalecer los servicios básicos y de asistencia.

2. Plan de Acción: Definición y puesta en marcha: Una vez desarrolladas las recomendaciones, diseñamos un Plan de Acción para su presentación a agentes políticos y sociales, interesados en adscribirse a las recomendaciones y trabajar en colaboración. Se desarrollaron las siguientes actividades:

- Identificación de agentes políticos y sociales. Elaboramos un directorio de instituciones públicas y privadas con capacidad de influencia política para incorporar dichas recomendaciones.

- Herramienta de Difusión. Redactamos y se publicó, con el apoyo de la organización «Data Center. Research for Justice», un informe que recogiera las motivaciones y resultados del diagnóstico comunitario y los resultados del mismo (Mujeres Unidas y Activas, 2009).

- Capacitación para dar presentaciones. Realizamos dos talleres para aquellas mujeres que habiendo participado en el proceso, quisieran formar parte de los comités de presentación de las recomendaciones.

- Comités de presentación de Informe. Se estableció un grupo de 10 mujeres que formaron dos equipos, uno para trabajar en la ciudad de Oakland, y otro para trabajar en San Francisco. Los grupos estuvieron formados por mujeres voluntarias y educadoras que habían sido parte del grupo motor.

- Priorización de interlocutores. Cada grupo elaboró un listado de organizaciones en las que presentar las recomendaciones en base a una 
serie de criterios: a) Non- profits y grupos comunitarios afines, para buscar formas de trabajo conjunto y preguntar por otras organizaciones (institucionales y comunitarias) que pudieran estar interesadas. b) Organizaciones comunitarias afines no conocidas, derivadas del grupo anterior c) instituciones o servicios específicos d) administraciones públicas y fundaciones privadas.

3. Implementación-Evaluación-Devolución. Mujeres Unidas y Activas inició el Plan de Acción, con una rueda de prensa en su sede de San Francisco a la que acudieron dos medios de comunicación local de habla hispana. A continuación comenzaron las reuniones con directores de Casas de Acogida, redes de colaboración entre servicios sociales y organizaciones comunitarias. En total se desarrollaron 40 presentaciones, destacando la realizada al Departamento del Estatus de la Mujer del Ayuntamiento de San Francisco, durante la cual Mujeres Unidas y Activas se vio reconocida por la importante labor de visibilización y promoción de los derechos del colectivo al que representa. En reacción a esto, algunas organizaciones, dedicadas a la provisión de servicios sociales en esta área, cuestionaron la identificación de la organización como un servicio propiamente de "violencia doméstica» partiendo de una definición asistencial. Durante estas reuniones MUA reivindicó su papel y trabajo por la mejora de la calidad de vida de muchas mujeres que, tal y como mostraba la investigación llevada a cabo, no se sienten identificadas en un gran número de servicios. Además incorporó muchas de las impresiones derivadas de las conversaciones establecidas en el diálogo interno de la propia organización, como por ejemplo, incorporar psicólogos infantiles y crear grupos de jóvenes, así como establecer protocolos de emergencia con servicios específicos.

\section{Resultados}

Esta investigación pone de manifiesto el potencial de procesos de IAP, no como un fin en sí mismo, sino como herramienta para el impulso de cambios que parten de la propia experiencia de quienes se involucran en el proceso. El alto nivel de compromiso con la intervención por parte de las mujeres de MUA estuvo promovido por su enfoque hacia el impulso de cambios concretos basados en sus necesidades y propuestas. Las herramientas metodológicas aplicadas sirvieron para facilitar la reflexión colectiva y definición de los pasos siguientes, incorporando la construcción de conocimiento junto con objetivos para la transformación de los dispositivos institucionales disponibles. Estos resultados contienen un impacto aún mayor si atendemos 
a tres niveles de diálogo interrelacionados. En primer lugar, encontramos un nivel de dialogo entre la mujer y el grupo de acogida. El proceso descrito permitió que aquellas necesidades identificadas por las participantes como individuales a través de, por ejemplo, la encuesta fueran trasladadas al ámbito grupal, a través de los grupos de discusión, permitiendo romper sensaciones de aislamiento a la vez que construir un análisis de problemáticas y necesidades que les afectaban como grupo. En segundo lugar, encontramos un nivel de diálogo dentro de propio grupo de Mujeres Unidas y Activas. La prevalencia de la atención individualizada, la falta de servicios de traducción en momentos clave y la asunción de la separación familiar como la «mejor»solución, fueron identificadas, entre otras cuestiones, por las participantes como formulas institucionales generalizadas que sin embargo no consiguen responder a sus necesidades y experiencias como mujeres migrantes, que no hablan inglés y que por las razones presentadas prefieren acudir a servicios comunitarios. Un tercer nivel de dialogo se desarrolló entre la organización y otros servicios trabajando en el mismo área de intervención, como grupos comunitarios y servicios institucionales específicos. De esta manera, la organización inició y afianzó relaciones con servicios sociales específicos estableciendo programas conjuntos de colaboración, como por ejemplo el acceso de mujeres capacitadas para el acompañamiento en Casas de Acogida. Así mismo, la presencia de Mujeres Unidas y Activas en espacios institucionales, como interlocutoras expertas, contribuyó a la promoción de servicios y programas basados en el empoderamiento y liderazgo en tanto que servicios de prevención y atención de violencia contra las mujeres.

\section{Discusión}

A menudo las mujeres que abogan por los derechos de quienes viven violencia de género se encuentran insertas en lógicas de victimización y desempoderamiento cuando buscan compromisos de cambio desde espacios institucionales (Coll, 2010). Sin embargo, a nivel local, estos pueden servir para impulsar procesos organizativos que incorporen las necesidades sentidas de la personas destinatarias de intervención (Pastor Seller, 2004, 2001). También pueden convertirse en escenarios de articulación ciudadana promovidos por quienes ponen en marcha definiciones de derechos propios orientados a la mejora de la calidad de vida colectiva. Para ello son necesarias herramientas de análisis estructural que nos permitan cambiar el enfoque institucional de victimización de las mujeres e incorporar intervenciones basadas en la capacidad resiliente de las personas, que potencien la autonomía en la toma de decisiones 
y reproduzca modelos de atención y acompañamiento (Fundación Cepaim, 2013; Wood \& Middleman, 1992). Como trabajadoras y trabajadores sociales la perspectiva estructural nos propone nuevas formas de intervención social a medida que la realidad se complejiza y surgen propuestas que impulsan políticas públicas de impacto más amplio y profundo (Sokoloff \& Dupont, 2005). Finalmente, abandonar la perspectiva de la violencia de género como un problema unidimensional, nos permite mejorar las respuestas al problema en la medida en que podamos desarrollar intervenciones adaptadas a las necesidades y realidades, culturales, políticas y económicas, de las mujeres con las que trabajamos.

Como trabajadoras y trabajadores sociales, casos como el que aquí se presentan nos ofrecen oportunidades para potenciar prácticas de Trabajo Social Comunitario vertebradas por organizaciones comunitarias. Este posicionamiento nos permite ser agentes activos en espacios de convergencia para incidir en problemas sociales, facilitando la incorporación de voces que a menudo están situadas en los márgenes de los espacios de toma de decisión.

Agradecimientos: La investigación aquí presentada no hubiera sido posible sin el trabajo y apoyo de las mujeres de la organización Mujeres Unidas y Activas: María de Jesús Jiménez, Juana Flores, Claudia Reyes, María Guadalupe Carrillo, Mari Chuy Hernández, Andrea Cristina Mercado, Carmen Denís, Andrea Lee y Ariana Gil Nafarrate. Así mismo, quiero hacer explícito mi agradecimiento a las mujeres participantes en los Comités Corazón y Clínicas del Alma de Oakland y San Francisco y a quienes dedicaron su tiempo, fuerza e historias de vida a la «Campaña de Derechos en Violencia Doméstica».

\section{Bibliografía}

Alberich NistAL, T. (2008). IAP, Redes y Mapas Sociales: Desde la investigación a la intervención social. Portularia, 8 (1), 131-151.

AlCAZAR-CAmpos, A. (2013). Social Work with Female Victims of Gender Violence: Analysis of an Experience in Southern Spain from a Feminist Perspective. Affilia, 28 (4), 366-378.

Alencar-Rodrigues, R. D, Cantera, L. M, y Strey, M. N. (2013). Violencia de género en la pareja contra mujeres inmigrantes: un estado del arte. Revista de Estudios de Género. LA VENTANA, 4 (37), 41-69.

ANDER-EGG, E. (2003). Repensando la investigación-acción-participativa. Lumen-Humanitas. 
Bhuyan, R., Mell, M., Senturia, K., Sullivan, M., y Shiu-Thornton, S. (2005). «Women Must Endure According to Their Karma» Cambodian Immigrant Women Talk About Domestic Violence. Journal of Interpersonal Violence, 20 (8), 902-921.

Bhuyan, R., y SEnturia, K. (2005). Understanding Domestic Violence Resource Utilization and Survivor Solutions among Immigrant and Refugee Women. Introduction to the Special Issue. Journal of Interpersonal Violence, 20 (8), 895-901.

COKER, D. (2006). Restorative justice, Navajo Peacemaking and domestic violence. Theoretical Criminology, 10 (1), 67-85.

Coll, K. (2010). Remaking Citizenship: Latina Immigrants and New American Politics. Stanford University Press.

CREnSHAW, K. W. (1991). Mapping the Margins: Intersectionality, Identity Politics, and Violence against Women of Color. Standford Law Review, 46 (6), 1241-1299.

FUNDACIÓN CEPAIM. (2013). Informe sobre Violencia de Género en Mujeres Inmigrantes. Grupo de análisis y trabajo sobre violencia de género en mujeres inmigrantes. Recuperado a partir de http://cepaim.org/th_gallery/ informe-sobre-violencia-de-genero-en-mujeres-inmigrantes/

De Genova, N. D. (2005). Working the Boundaries: Race, Space, and «Illegality» in Mexican Chicago. Duke University Press.

Greenwood, D. J., y Levin, M. (2006). Introduction to Action Research: Social Research for Social Change. SAGE Publications.

Greenwood, D. J., Whyte, W. F., y Harkavy, I. (1993). Participatory Action Research as a Process and as a Goal. Human Relations, 46 (2), 175-192.

Humphries, B. (2008). Social Work Research for Social Justice. Palgrave Macmillan. ingram, M., McClelland, D. J., Martin, J., Caballero, M. F., Mayorga, M. T., y GiLlespie, K. (2010). Experiences of Immigrant Women Who Self-Petition Under the Violence Against Women Act. Violence Against Women, 16 (8), 858-880.

IOÉ, C. (1993). Investigación-acción participativa. Introducción en España. Documentación social, 92, 59-70.

LEE, J. A. B. (2013). The Empowerment Approach to Social Work Practice. Columbia University Press.

Menjívar, C., y Salcido, O. (2002). Immigrant Women and Domestic Violence. Common Experiences in Different Countries. Gender \& Society, 16 (6), 898-920.

Merry, S. E. (2009). Human Rights and Gender Violence: Translating International Law into Local Justice. University of Chicago Press.

Minkler, M. (2005). Community Organizing and Community Building for Health. Rutgers University Press. 
Minkler, M., y Wallerstein, N. (2011). Community-Based Participatory Research for Health: From Process to Outcomes. John Wiley \& Sons.

Montañez, P., y Moyano, M. (2006). Violencia de género sobre inmigrantes en España. Un análisis psicosocial. Pensamiento psicológico, 2 (6), 21-32.

MUJERES UNIDAS Y ACTIVAS. (2009). Ecos del Silencio, Levantando Nuestra Voz. San Francisco, Oakland. Recuperado a partir de http://www.datacenter. org/wp-content/uploads/Mua-English-report.pdf

PAStor Seller, E. (2001). Iniciativa social y trabajo social comunitario. Alternativas. Cuadernos de Trabajo Social, 9, 169-191.

PASTOR SEller, E. (2004). La participación ciudadana en el ámbito local, eje transversal del trabajo social comunitario. Alternativas. Cuadernos de Trabajo Social, 12, 103-137.

RiverA, J. (1994). Doméstic Violence Against Latinas by Latino Males: An Analysis of Race, National origin, and Gender Differentials. Boston College Third World Law Journal, 14 (2), 231-258.

Shiu-Thornton, S., Senturia, K., y Sullivan, M. (2005). «Like a Bird in a Cage» Vietnamese Women Survivors Talk About Domestic Violence. Journal of Interpersonal Violence, 20 (8), 959-976.

Sokoloff, N. J., y Dupont, I. (2005). Domestic Violence at the Intersections of Race, Class, and Gender Challenges and Contributions to Understanding Violence Against Marginalized Women in Diverse Communities. Violence Against Women, 11 (1), 38-64.

Strang, H., y BraithWAite, J. (2002). Restorative Justice and Family Violence. Cambridge University Press.

Sullivan, M., Bhuyan, R., Senturia, K., Shiu-Thornton, S., y Ciske, S. (2005). Participatory Action Research in Practice A Case Study in Addressing Domestic Violence in Nine Cultural Communities. Journal of Interpersonal Violence, 20 (8), 977-995.

Tudela-VÁzquez, M. P. (2013). «Ecos del Silencio, Levantando Nuestra Voz»: traducciones locales de los Derechos Humanos de las Mujeres. Trabajo Social Global-Global Social Work, 3 (4), 113-132.

Villalon, R. (2010). Violence Against Latina Immigrants: Citizenship, Inequality, and Community. NYU Press.

Villasante, T., y Montañés Serrano, M. (2000). Algunos cambios de enfoque en las ciencias sociales. La investigación social participativa. En T. Villasante, M. Montañés Serrano, y J. Martí, La investigación social participativa. Construyendo Ciudadanía. (2002. ${ }^{a}$ ed.). Vol. 1, 14-60. Madrid: El Viejo Topo.

WAllerstein, N. (2000). A participatory evaluation model for Healthier Communities: developing indicators for New Mexico. Public Health Reports, 115 (2-3), 199-204. 
Wood, G. G., y Middleman, R. R. (1992). Groups to Empower Battered Women. Affilia, 7 (4), 82-95.

Yoshinama, M. (1999). Domestic Violence Against Women of Japanese Descent in Los Angeles: Two Methods of Estimating Prevalence. Violence Against Women, 5 (8), 869-897.

\section{ANTECEDENTES DE DIFUSIÓN}

Investigación basada en el trabajo de campo desarrollado para la tesis doctoral «La Organización de Nosotras: Procesos de ciudadanía a partir de experiencias de 'ilegalidad' en Estados Unidos. Aprendizajes con Mujeres Unidas y Activas»

Alternativas. Cuadernos de Trabajo Social, 22, 2015, pp. 185-206 - ISSN 1133-0473 